\title{
CS-15 Impacto en la sobrevivencia del inicio programado de diálisis peritoneal (DP) en pacientes con enfermedad renal crónica (ERC)
}

Impact on patient survival of on scheduled initiation of peritoneal dialysis $(P D)$ program in chronic kidney disease patients (CKD)

\author{
Erick F. Herrera, Alejandro Lucas, Vicente Sánchez-Polo*
}

Departamento de Nefrología y Trasplante Renal, Instituto Guatemalteco de Seguridad Social (IGSS), Guatemala.

*Autor al que se dirige la correspondencia: visanpolo@gmail.com

\section{Resumen}

E 1 IGSS es la principal institución de atención de pacientes con ERC en Guatemala. En 2009 se inició el Programa de Salud Renal (PSR) para detectar, prevenir y retardar la progresión de la ERC y referir a los pacientes al mejor tratamiento disponible (DP, hemodiálisis o trasplante). El estudio se hizo para determinar si el PSR tiene impacto en la supervivencia de los pacientes que ingresan de manera programada. Se realizó un estudio descriptivo, longitudinal en pacientes que ingresaron al programa de DP de enero 2012 a julio 2013. Los de inicio programado fueron vistos tres veces en la Clínica de Salud Renal y los no programados llegaron de emergencia al Hospital de Enfermedades; a todos se dio seguimiento por un año. Se revisaron expedientes y laboratorios y se aplicaron promedios, prueba de $\chi^{2}$ y curvas de supervivencia. Se incluyeron 169 pacientes, 58 programados y 111 de urgencia. No existen diferencias entre grupos en las características basales. Según la Curva de Kaplan-Meier hay supervivencia significativa en el grupo de inicio programado (89.6\%) vr los de emergencia (73.8\%) (p .015). No hubo significancia al año en cuanto a calidad de diálisis expresada por el modelo matemático de KT/V (66 vr $59.5 \%, \mathrm{p}=.450)$, peritonitis $(22.4 \mathrm{vr} 21.6 \%, \mathrm{p}=.787)$ o cambio a hemodiálisis $(10.3 \mathrm{vr} 9.9 \%, \mathrm{p}=.968)$. El inicio programado de DP tiene un impacto positivo en la supervivencia de los pacientes. No existen diferencias en los demás marcadores de calidad de diálisis luego de un año de seguimiento.

Palabras claves: Patología renal, inicio programado, supervivencia

\begin{abstract}
$\mathrm{T}$ he Guatemalan Social Security Institute (IGSS) is the main provider of medical care to patients with CKD in Guatemala. In July 2009, the renal health program (RHP) was initiated to determine whether the RHP has also impacted the quality of PA, outcomes between those enrolled in scheduled vr unscheduled was compared. A longitudinal, descriptive study was performed among patients enrolled in PD from January 2012 to July 2013, scheduled patients were those who had at least three prior consultation at the RHP, not scheduled were those who started dialysis urgently, all of whom were followed for one year. Using chart reviews express the results in averages, percentages, $\chi^{2}$ test, and survival curves. Data from 169 patients was obtained, 58 enrolled in a scheduled manner while 111 were not. There were no significant differences in baseline characteristics between the groups. Using Kaplan-Meier plots, there was a significant survival advantage among those enrolled in scheduled PA $89.6 \% \mathrm{vr}$ $73.8 \%(\mathrm{p}=.015)$. There were no significant differences between groups in obtaining goal $\mathrm{Kt} / \mathrm{V}(66 \% \mathrm{vr} 59.5 \%$, $\mathrm{p}$ $=.40)$, incidence of peritonitis $(22.4 \%$ vr $21.6 \%, \mathrm{p}=.787)$, or change to hemodialysis $(10.3 \%$ vr $9.9 \%, \mathrm{p}=.968)$. There is a clear impact on patient survival between those enrolled in scheduled vr non-scheduled PD through the IGSS. There were no differences between other markers of dialysis quality.
\end{abstract}

